\title{
Microscopic quantification of bacterial invasion by a novel antibody-independent staining method
}

\author{
Franziska Agerer, Stephanie Waeckerle, Christof R. Hauck* \\ Zentrum für Infektionsforschung, Universität Würzburg, Röntgenring 11, 97070 Würzburg, Germany
}

Received 31 March 2004; received in revised form 18 May 2004; accepted 21 May 2004

\begin{abstract}
Microscopic discrimination between extracellular and invasive, intracellular bacteria is a valuable technique in microbiology and immunology. We describe a novel fluorescence staining protocol, called FITC-biotin-avidin (FBA) staining, which allows the differentiation between extracellular and intracellular bacteria and is independent of specific antibodies directed against the microorganisms. FBA staining of eukaryotic cells infected with Gram-negative bacteria of the genus Neisseria or the Grampositive pathogen Staphylococcus aureus are employed to validate the novel technique. The quantitative evaluation of intracellular pathogens by the FBA staining protocol yields identical results compared to parallel samples stained with conventional, antibody-dependent methods. FBA staining eliminates the need for cell permeabilization resulting in robust and rapid detection of invasive microbes. Taken together, FBA staining provides a reliable and convenient alternative for the differential detection of intracellular and extracellular bacteria and should be a valuable technical tool for the quantitative analysis of the invasive properties of pathogenic bacteria and other microorganisms.
\end{abstract}

Keywords: Bacterial invasion; Intracellular bacteria; Fluorescence staining; Neisseria gonorrhoeae; Staphylococcus aureus

\section{Introduction}

A common theme that has emerged from the study of infectious diseases at the cellular level is the pathogen-induced entry into eukaryotic cells, a process termed bacterial invasion (Falkow, 1991; Finlay and Cossart, 1997). Examples of potentially invasive bacteria include Legionella pneumophila, Listeria

* Corresponding author. Tel.: +49-931-312137; fax: +49-931312578 .

E-mail address: christof.hauck@mail.uni-wuerzburg.de (C.R. Hauck). monocytogenes, Mycobacterium tuberculosis, Neisseria gonorrhoeae, Salmonella typhimurium, Shigella flexneri or Staphylococcus aureus. In the case of $N$. gonorrhoeae, invasion can be mediated by neisserial colony opacity-associated (Opa) proteins binding to receptors of the CEACAM family present on human cells (see, for review, Hauck and Meyer, 2003). In the case of $S$. aureus, invasion is triggered by fibronectin recruitment to the surface of these Gram-positive microbes. Fibronectin then serves as a molecular bridge to allow bacterial attachment to host $\beta 1$ integrins (Sinha et al., 1999; Fowler et al., 2000; Agerer et al., 2003). To analyse bacterial invasion in vitro and to 
quantify the invasive potential of pathogens, two experimental approaches have been used extensively. Antibiotic protection assays on the one hand allow the convenient quantification of intracellular bacteria. However, as this sort of assay is based on the recovery of viable bacteria after their internalisation by eukaryotic cells, it does not discriminate between efficient bacterial invasion in combination with low intracellular survival or low invasiveness coupled to effective intracellular survival (Booth et al., 2003). Therefore, such assays have to be complemented with microscopic evaluation of infected samples. To allow the differentiation between attached, but extracellular and bona fide intracellular microorganisms, an elaborate immunofluorescence staining protocol has been developed (Heesemann and Laufs, 1985) and is in general use (Dehio et al., 1997; Williams et al., 1998; Luhrmann et al., 2001). This double cycle antibody (AB) staining protocol takes advantage of the intact host cell membrane as it is impermeable for antibodies. Accordingly, extracellular bacteria are stained in a first cycle with a bacteria-specific primary and a dye-coupled secondary antibody (e.g., FITCcoupled). Then, the host cell membrane is permeabilized by detergent or methanol treatment and the intracellular bacteria are now accessible for a second cycle of staining with the same primary, but a different secondary antibody coupled to a distinct fluorescent dye (e.g., Cy-5). As a result, extracellular bacteria will be labelled by both fluorescent dyes (FITC and Cy-5), whereas intracellular bacteria will only be marked by the fluorescent dye used in the second staining cycle (in our example in Fig. 1, Cy-5). Samples prepared according to this double cycle $\mathrm{AB}$ staining procedure allow the precise determination of intracellular bacteria at any given moment during the invasion process. However, as this protocol relies on multiple cycles of immunofluorescence staining, it not only requires considerable amounts of specific antibodies against the microbes, but it is also time consuming.

Here, we report on a novel staining procedure to discriminate between extracellular and intracellular bacteria that is independent of specific antibodies to detect the microorganisms. The independence from antibodies to detect the bacteria makes this method particularly useful in situations, where specific antisera are not (yet) available. In addition, as this protocol, in contrast to the double cycle AB staining, requires only a single staining cycle, it is less laborious and saves valuable resources compared to common approaches. This novel and fast method allows the quantitative evaluation of intracellular bacteria and should be a valuable technique in the areas of cellular microbiology and immunology.

\section{Materials and methods}

\subsection{Cell culture and transfection}

The human embryonic kidney cell line 293T (293 cells) was grown in DMEM $/ 10 \%$ calf serum (CS) at $37{ }^{\circ} \mathrm{C}, 5 \% \mathrm{CO}_{2}$. Mouse embroyo fibroblasts were cultured as described (Sieg et al., 1999). Cells were subcultured every 3-4 days. Transfection of 293 cells was accomplished with standard calcium-phosphate co-precipitation using $5 \mu \mathrm{g}$ plasmid $/ 10 \mathrm{~cm}$ culture dish. Cells were used 2 days after transfection. In some cases, cells were treated $5 \mathrm{~min}$ prior to infection with $1 \mu \mathrm{g} / \mathrm{ml}$ cytochalasin D or $1 \mu \mathrm{M}$ PP2 (Calbiochem, Schwalbach, Germany) and inhibitors remained with the cells throughout the infection.

\subsection{Bacteria}

$\mathrm{Opa}_{\mathrm{CEA}}$-expressing $\left(\mathrm{Opa}_{52}\right)$, non-piliated N. gonorrhoeae MS11-B2.1 (strain N309) and Opa-negative, non-piliated N. gonorrhoeae MS11-B1 (strain N302) were obtained from T. Meyer (MPI Infektionsbiologie, Berlin, Germany) and grown at $37{ }^{\circ} \mathrm{C}, 5 \% \mathrm{CO}_{2}$ on GC agar (Gibco BRL, Praisley, UK) supplemented with vitamins and the appropriate antibiotics.

S. aureus (strain Cowan) and Staphylococcus carnosus were cultured in Tryptic Soybean Broth (TSB; BD Biosciences, Heidelberg, Germany) at $37{ }^{\circ} \mathrm{C}$ and harvested in a mid-logarithmic phase.

\subsection{Biotinylation and labelling of bacteria}

Bacteria $\left(1 \times 10^{9} / \mathrm{ml}\right)$ were washed twice with sterile phosphate-buffered saline (PBS), suspended in $0.4 \mu \mathrm{g} / \mathrm{ml} \mathrm{5-(6)-carboxyfluorescein-succinylester}$ (Molecular Probes, Eugene, OR) dissolved in PBS (FITC-PBS), and incubated for 15 min under constant shaking at $4{ }^{\circ} \mathrm{C}$. Sulfo-NHS-LC-biotin (Perbio Science, Bonn, Germany) was solved at a final concen- 


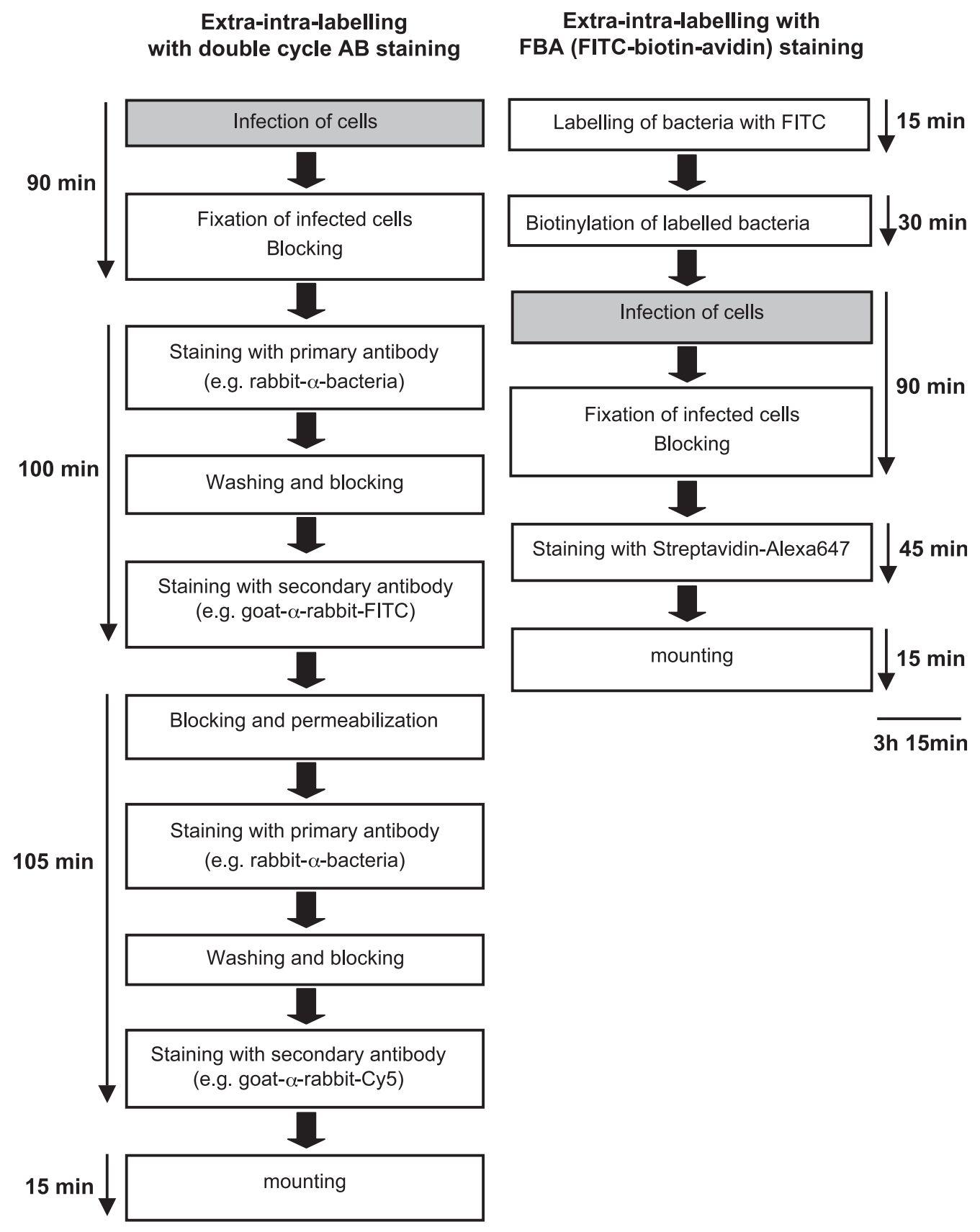

\section{$5 \mathrm{~h} 10 \mathrm{~min}$}

Fig. 1. Schematic workflow of the staining protocols employed. The left column summarizes the conventional double cycle antibody (AB) labelling protocol. The right column provides an overview of the antibody-independent FITC-biotin-avidin (FBA) staining procedure. The time bars at the margins indicate the recommended schedule. 
tration of $0.3 \mathrm{mg} / \mathrm{ml}$ in PBS. Identical volumes of bacteria in FITC-PBS and the biotin solution were combined and further incubated for $30 \mathrm{~min}$ at $4{ }^{\circ} \mathrm{C}$. Next, biotinylated and FITC-labelled bacteria were washed three times with PBS and resuspended in PBS with $1 \%$ bovine albumin prior to use.

\subsection{Infection of the cells}

Transfected 293 cells were seeded on glass coverslips in 24-well plates (coated with a combination of fibronectin $(4 \mu \mathrm{g} / \mathrm{ml})$ and poly-L-lysine $(10 \mu \mathrm{g} / \mathrm{ml})$ in PBS) in regular cell culture medium (DMEM/10\% CS) 1 day before infection. For infection, Neisseria were taken from plates and staphylococci were harvested by centrifugation. Bacteria were washed, resuspended in PBS, labelled with FITC and with or without biotin, and applied to cells at a multiplicity of infection of 40 for $1 \mathrm{~h}$ (Neisseria) or $2 \mathrm{~h}(S$. aureus). Infected samples were fixed for $20 \mathrm{~min}$ with $4 \%$ paraformaldehyde in PBS, washed three times with PBS and blocked with PBS containing $10 \%$ CS (blocking buffer) for $5 \mathrm{~min}$ at room temperature before further processing.

\subsection{Double cycle AB staining}

An outline of the procedure is shown in Fig. 1. After blocking of fixed samples, extracellular bacteria were stained for $45 \mathrm{~min}$ with suitable dilutions of polyclonal rabbit- $\alpha-N$. gonorrhoeae MS11 (1:200 of AK92; MPI für Biologie, Berlin) in blocking buffer at room temperature. Samples were washed twice with PBS, blocked again for 5 min with blocking buffer and incubated for 45 min with Cy5-conjugated goat$\alpha$-rabbit IgG (1:100; Jackson ImmunoResearch, West Grove, PA) at room temperature. After two washes with PBS, samples were incubated for 5 min with PBS containing $10 \% \mathrm{CS}$ and $0.2 \%$ saponin (permeabilization buffer) to permeabilize cellular membranes. Cells were again incubated with polyclonal rabbit- $\alpha-N$. gonorrhoeae MS11 (1:200 of AK92; MPI für Infektionsbiologie, Berlin) in blocking buffer for $45 \mathrm{~min}$ at room temperature, washed two times with PBS, incubated in permeabilization buffer for $5 \mathrm{~min}$ and stained for 45 min with Cy2-conjugated goat- $\alpha$-rabbit IgG (1:100; Jackson ImmunoResearch). After three washes with PBS, samples were mounted in embed- ding medium (Dako, Glostrup, Denmark) on glass slides and sealed with nail polish.

\subsection{Single cycle FITC-biotin-avidin (FBA) staining}

An outline of the procedure is shown in Fig. 1. Cells infected with biotinylated and FITC-labelled bacteria were fixed and blocked as described above. After blocking, extracellular bacteria were stained for $45 \mathrm{~min}$ at room temperature with StreptavidinAlexa647 (Molecular Probes) diluted 1:200 in blocking buffer. Where indicated, the actin cytoskeleton of the eukaryotic cells was counterstained with AlexaFluor-546 phalloidin (phalloidin-Red; Molecular Probes) for $30 \mathrm{~min}$ after $5 \mathrm{~min}$ incubation in permeabilization buffer. Samples were washed three times with PBS, mounted with embedding medium (Dako) on glass slides and sealed with nail polish.

\subsection{Immunofluorescence microscopy and imaging}

Samples were viewed with an LSM 510 confocal laser scanning microscope (Zeiss, Oberkochen, Germany) using a $40 \times, 1.3$ NA Plan Neofluar oilimmersion objective. Fluorescence signals of doubleand triple-labelled specimens were serially recorded with appropriate excitation and emission filters to avoid bleed-through. Images were digitally processed with Photoshop 6.0 (Adobe Systems, Mountain View, $\mathrm{CA}$ ) and merged to yield pseudo-coloured pictures.

\section{Results}

\subsection{Double cycle AB staining to detect intracellular N. gonorrhoeae}

To analyse the CEACAM-mediated internalisation of $N$. gonorrhoeae by fluorescence microscopy, CEACAM-negative human 293 cells were transfected with cDNA encoding CEACAM3 fused to red fluorescent protein (CEACAM3-RFP) and samples were fixed for $1 \mathrm{~h}$ following infection with gonococci. Previously, we have observed that 293 cells transfected with the empty expression vector do not support Opa-mediated invasion of $N$. gonorrhoeae within this time frame (Schmitter et al., 2004). Fixed samples were further processed according to the conventional double cycle 
antibody staining protocol that allows the discrimination between extracellular and intracellular microorganisms (double cycle $\mathrm{AB}$ staining; Fig. 1). As a result, Opa-expressing gonococci could be detected that were positive for the fluorescent dye employed after permeabilization of the cells, but did not produce a signal with the dye employed in the first staining cycle demonstrating their intracellular localization (Fig. 2A; small arrows). As expected, in samples infected with non-opaque gonococci all bacteria were marked as being extracellular indicating that this staining method correctly discriminates between extracellular and intracellular bacteria. Quantification of the samples revealed that in the case of Opa-positive $N$. gonorrhoeae about $56 \%$ of the cell-associated bacteria were located intracellularly in CEACAM3expressing 293 cells already $1 \mathrm{~h}$ after the infection (Fig. 2B,C). Clearly, bacteria lacking a CEACAMbinding Opa protein were not internalised (Fig. 2B,C). Though the enumeration of parallel samples demonstrated the reproducibility of this method, the staining intensity was dependent on the quality of the antiserum and the proper permeabilization of the host cell membrane and sometimes varied from experiment to experiment (Agerer and Hauck, unpublished observations). In addition, due to the double cycle of immunofluorescence staining, the procedure was time consuming (Fig. 1). Therefore, we established a novel staining protocol that does not require specific antibodies or permeabilization of the infected cells and that has a reduced time requirement.

\subsection{Detection of intracellular bacteria by FBA staining}

As the novel protocol relies on the use of FITC, biotin and avidin, we have termed this procedure FBA staining. For FBA staining, gonococci were covalently labelled prior to infection with 5-(6)-carboxyfluorescein-succinylester (FITC) and simultaneously biotinylated with sulfo-NHS-LC-biotin (biotin) (Fig. 1). Following infection and fixation, samples were subjected to a single round of staining using Streptavidin-Alexa647 without permeabilization of the cells. As a result, both extracellular and intracellular bacteria should appear FITC-positive, whereas only the extracellular bacteria should be labelled with Alexa647. To allow a comparison of the new staining procedure with the conventional double cycle $\mathrm{AB}$ staining, we employed the same batch of CEACAM3-RFP transfected 293 cells as detailed in Section 3.1 in our novel staining protocol. When CEACAM3-RFP-expressing and Neisseria-infected 293 cells were processed according to the FBA staining protocol, we again could easily distinguish between extracellular and intracellular bacteria (Fig. 2A). As observed before, intracellular bacteria were only detected when cells were infected with Opaexpressing gonococci, whereas non-opaque bacteria were exclusively located outside the cells (Fig. 2A). In addition, the fluorescence signal emitted from the covalently labelled bacteria was stronger than the signal observed upon double cycle $\mathrm{AB}$ staining making detection of the bacteria and discrimination between extracellular and intracellular localization an easy task (compare lower panels in Fig. 2B). Importantly, quantification of intracellular bacteria using the FBA staining protocol gave nearly identical results (58\% intracellular bacteria) compared to the more laborious double cycle $\mathrm{AB}$ staining (Fig. 2B,C), demonstrating that the novel method is a valid alternative to established procedures. It also indicated that the covalent modification of the bacterial surface with FITC and biotin prior to infection did not influence the CEACAM-mediated internalisation of Opaexpressing $N$. gonorrhoeae.

\subsection{Actin dynamics and Src family kinases are critical for the CEACAM3-mediated uptake of Opa-expressing N. gonorrhoeae}

To validate our results further, we applied the FBA staining procedure to samples, where the CEACAM3expressing cells were treated with pharmacological inhibitors prior to the infection with Opa-expressing $N$. gonorrhoeae. Both the actin depolymerizing agent cytochalasin D as well as the Src family kinase inhibitors have been shown to strongly interfere with CEACAM3-mediated internalisation (Hauck et al., 1998; Billker et al., 2002; McCaw et al., 2003; Schmitter et al., 2004). As observed before, numerous intracellular gonococci could be detected in CEACAM3-transfected cells following the FBA staining (Fig. 2D). Co-staining of filamentous actin by phalloidin-Red demonstrated that treatment of the cells with cytochalasin D or the Src family kinase inhibitor 


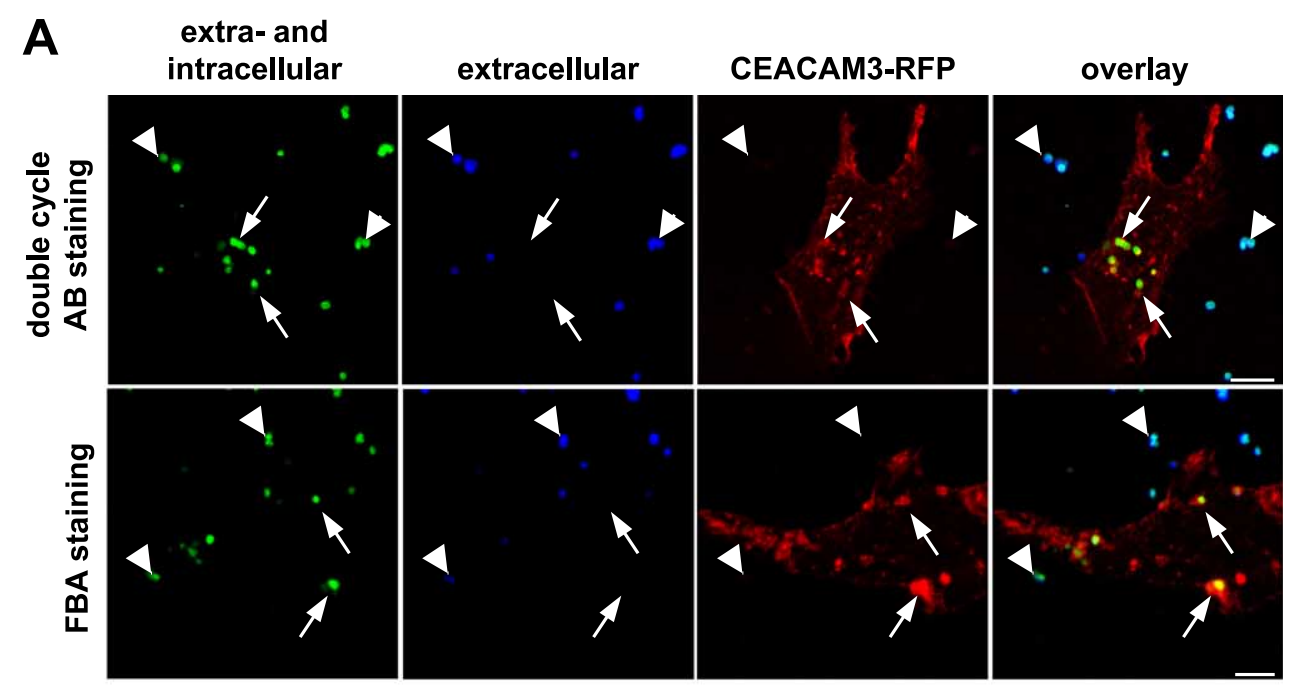

B

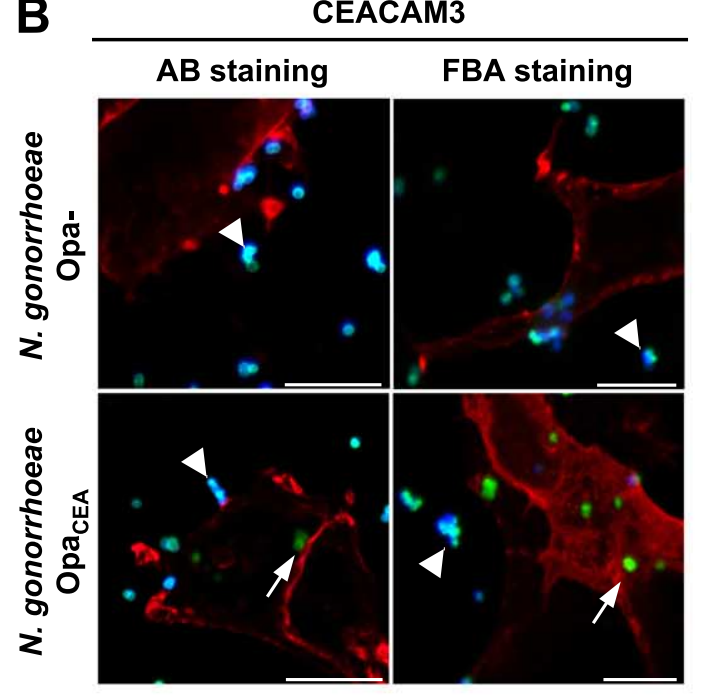

C

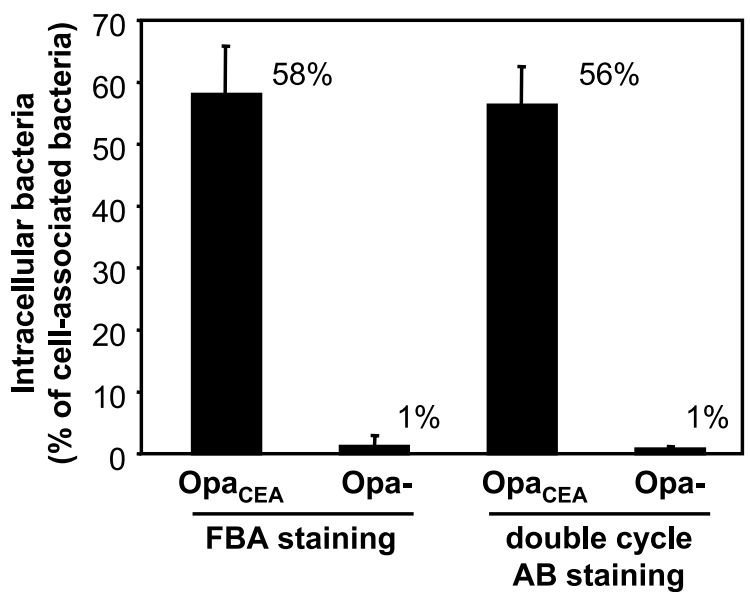

D

N. gonorrhoeae Opa $\mathrm{CEA}_{\mathrm{C}}$

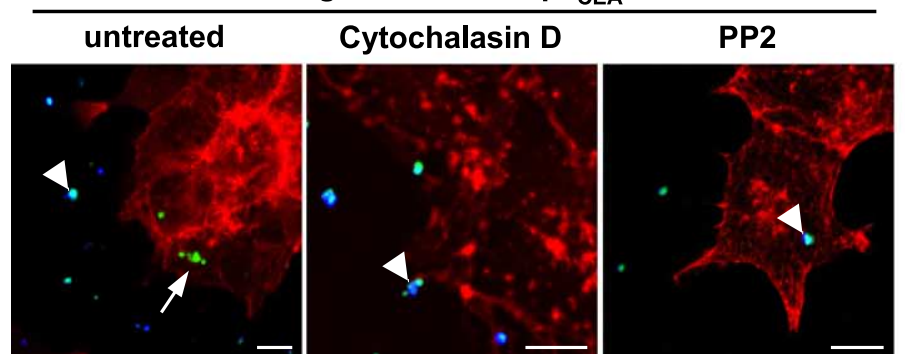


PP2 not only disrupted the proper organization of the actin cytoskeleton, but also completely abrogated the uptake of Opa-expressing N. gonorrhoeae (Fig. 2D). Importantly, FBA staining clearly revealed that all cell-associated gonococci were located extracellularly, when cells were treated with either PP2 or cytochala$\sin \mathrm{D}$ (Fig. 2D).

\subsection{FBA staining to evaluate the invasive potential of commensal Neisseria}

Since FBA staining is independent of antibodymediated labelling, it should be of particular use in situations where the invasive potential of bacterial strains or species is to be evaluated, but where specific antibodies against these microorganisms are not readily available. Accordingly, we applied the FBA staining protocol to evaluate the CEACAM-mediated internalisation of several commensal Neisseria species that were not recognized by our polyclonal antiserum raised against $N$. gonorrhoeae strain MS11 (data not shown) and where commercial antibodies are not available. Compared to the invasive Opa-expressing $N$. gonorrhoeae, all four tested commensal species, Neisseria mucosa, Neisseria lactamica, Neisseria cinerea and Neisseria sicca, were not internalised by the CEACAM-expressing 293 cells (Fig. 3A, data not shown). Accordingly, all commensal bacteria in the infected samples were labelled by both FITC and Streptavidin-Alexa647, whereas a major portion of the opaque gonococci was labelled only by FITC indicating their intracellular localization (Fig. 3A). These results demonstrated that the novel FBA staining procedure not only allows fast and clear-cut differentiation between intracellular and extracellular bacteria, but should also be applicable to a large variety of bacteria where specific antibodies are not readily available.

\subsection{FBA staining of Gram-positive S. aureus}

In order to investigate the applicability of the FBA staining procedure to bacteria other than Gram-negative Neisseria, we choose to monitor invasion of $S$. aureus into human cells. Previously, we and others have demonstrated that $S$. aureus is able to exploit fibronectin and host cell $\beta 1$ integrins to gain access to an intracellular compartment, whereas the related nonpathogenic species $S$. carnosus does not invade eukaryotic cells (Sinha et al., 1999; Fowler et al., 2000; Agerer et al., 2003). Therefore, we labelled both species of staphylococci with FITC and biotin and then infected 293 cells for $2 \mathrm{~h}$. Again, processing of the fixed samples according to the FBA staining procedure resulted in a clear discrimination between extracellular and intracellular bacteria (Fig. 3B). In particular, a large fraction of $S$. aureus was observed to be located within the human cells and therefore was not detected by Streptavidin-Alexa647 (Fig. 3B, small arrows). In addition, co-staining with phalloidin-Red revealed accumulation of polymerised actin at the site of bacterial attachment (Fig. 3B; double arrowhead). In contrast to $S$. aureus, the non-pathogenic S. carnosus was exclusively located on the outside of the cells and did not stimulate actin polymerisation (Fig. 3B). A similar pattern of invasiveness has been observed before using antibiotic protection assays and antibody-dependent staining procedures (Agerer et al., 2003), indicating that the covalent modification of $S$. aureus by FITC and biotin does not impair the integrin-mediated internalisation process. Taken together, these results demonstrate that the novel antibody-independent FBA

Fig. 2. Comparison between the conventional double cycle AB staining and FBA staining with regard to differential labelling of extracellular

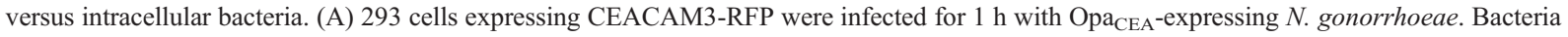
were either labelled with FITC and biotin prior to infection (FBA staining; lower row) or used unlabelled (upper row). As indicated, samples were processed according to the double cycle AB staining or the FBA staining protocols, respectively. Extracellular (arrowhead) and intracellular bacteria (small arrow) could be easily distinguished by both staining procedures according to their differential staining pattern. (B) CEACAM3-RFP-expressing 293 cells were infected for $1 \mathrm{~h}$ with $\mathrm{Opa}_{\mathrm{CEA}}$-expressing (Ngo Opa $\mathrm{CEA}_{\text {) }}$ or non-opaque (Ngo Opa-) $N$. gonorrhoeae and processed according to either the double cycle AB staining or the FBA staining. Examples of extracellular (arrowhead) and intracellular bacteria (small arrow) are indicated. (C) Samples prepared as in (B) were evaluated for intracellular and extracellular bacteria. Bars represent the mean percentage intracellular bacteria of the total cell-associated bacteria (100 infected cells in random fields of triplicate samples were counted) from four independent experiments \pm standard deviation. (D) 293 T cells were transfected with CEACAM3. Cells were treated with $1 \mu \mathrm{g} / \mathrm{ml}$ cytochalasin D or $1 \mu \mathrm{M}$ PP2 and infected with Opa ${ }_{\mathrm{CEA}} N$. gonorrhoeae for $1 \mathrm{~h}$. Fixed cells were stained for extracellular (arrowhead) and intracellular bacteria (small arrow) according to the FBA staining protocol and polymerised actin was visualized with phalloidin-Red. The scale bars represent $10 \mu \mathrm{m}$. 


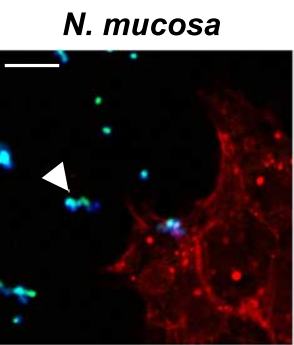

\section{N. lactamica}

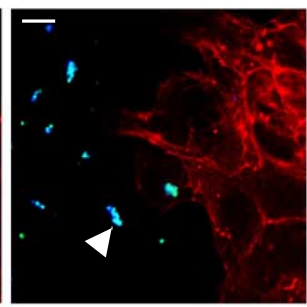

phalloidin-Red
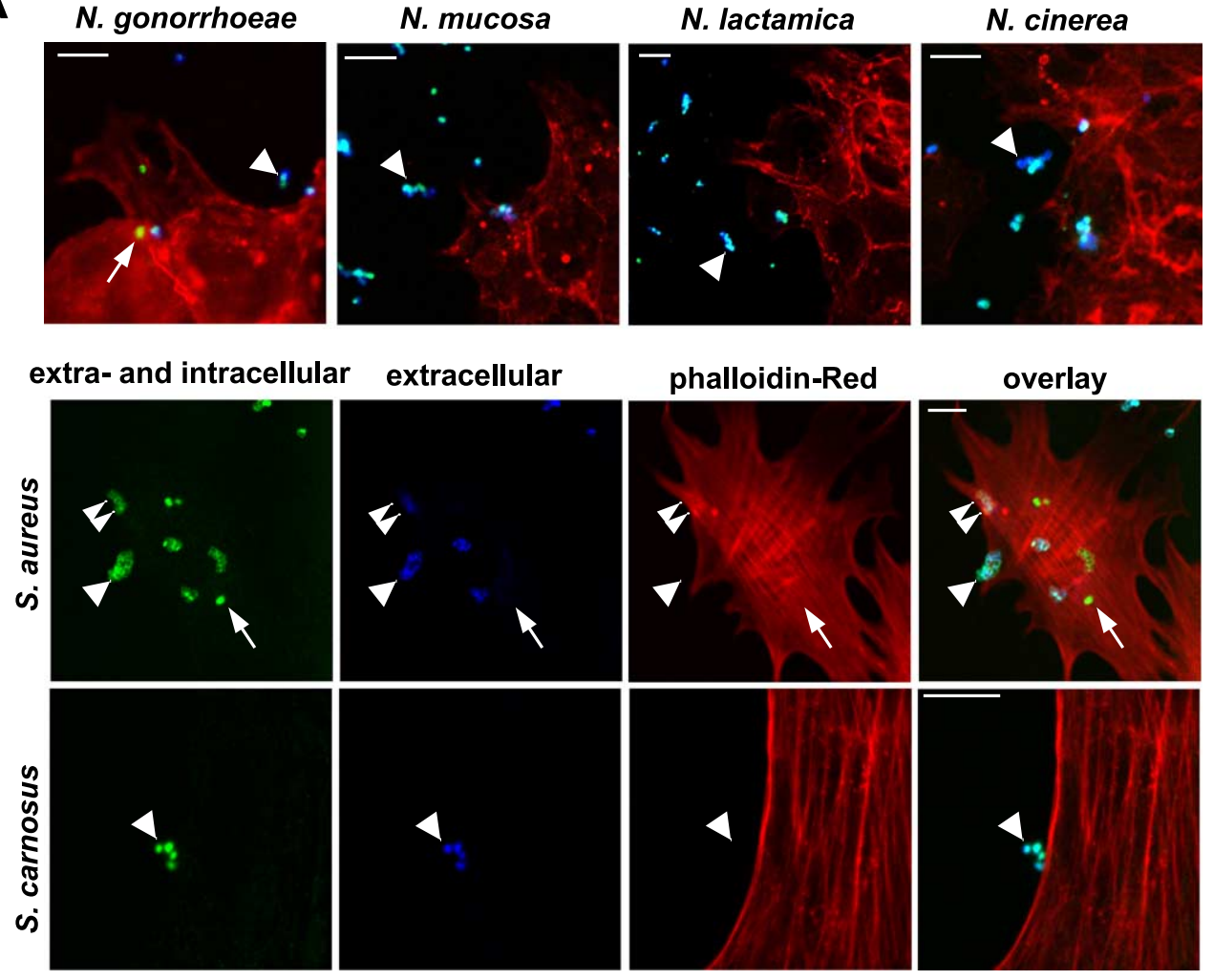

Fig. 3. Application of the FBA staining to analyse the invasive potential of commensal Neisseria and Gram-positive staphylococci. (A) 293 cells were transfected with RFP-CEACAM3. After 2 days, cells were infected for $1 \mathrm{~h}$ with FITC- and biotin-labelled commensal Neisseria mucosa, $N$. cinerea, $N$. lactamica or pathogenic Opa ${ }_{\mathrm{CEA}}$-expressing $N$. gonorrhoeae at a MOI of 30 . Samples were processed according to the FBA staining protocol to distinguish between extracellular (arrowhead) and intracellular (small arrow) bacteria. Additionally, the actin cytoskeleton of the cells was counterstained by phalloidin-Red. (B) Mouse fibroblasts were infected with the invasive $S$. aureus (Cowan) and the nonpathogenic $S$. carnosus for $2 \mathrm{~h}$ at a MOI 40. Fixed samples were labelled according to the FBA staining protocol for extracellular (arrowhead) and intracellular bacteria (small arrow). The actin cytoskeleton of the cells was counterstained by phalloidin-Red. Actin polymerisation in the vicinity of cell-attached $S$. aureus can be observed (double arrowhead). Bars represent $10 \mu \mathrm{m}$.

staining procedure is a convenient and reliable technique for distinguishing between extracellular and intracellular bacteria. In addition, this method is applicable to a wide range of pathogen-host cell sys-tems and therefore allows comparative investigations.

\section{Discussion}

Discrimination between extracellular and intracellular organisms is an important pre-requisite to estimate the invasiveness of microbes. Accordingly, several microscopic methods have been developed to accomplish this task (Heesemann and Laufs, 1985;
Drevets and Campbell, 1991; Drevets and Elliott, 1995). Here, we present a novel staining procedure that takes advantage of bacteria simultaneously labelled with FITC and biotin. The four main features of this novel procedure are: (i) the antibody independence; (ii) the robust and specific signals obtained; (iii) no need for permeabilization of the infected cells; and (iv) the convenient and fast protocol.

The obvious advantage of the FBA staining procedure is its independence from specific antibodies usually needed to detect bacteria. This might be of particular importance in situations where antisera against the microorganism of interest are not readily available. This feature allowed us to microscopically 
analyse for the first time the CEACAM3-mediated recognition and internalisation of commensal Neisseria. The lack of intracellular commensal $N$. mucosa, $N$. lactamica, $N$. cinerea and $N$. sicca as observed by the FBA staining protocol demonstrates that these species are not taken up by the human host cells via this receptor. Therefore, FBA staining might be a useful tool to investigate bacteria-host interactions with regard to receptor-mediated uptake processes.

Instead of using a specific antiserum in each case, the FBA staining protocol allows the application of identical reagents to monitor the uptake of diverse bacteria. As demonstrated in this report with a wide selection of Gram-negative and Gram-positive bacterial species, FBA staining not only saves resources, but also allows a direct comparison between the different samples that is otherwise often hampered by different qualities of antiserum. The direct labelling of the bacteria also circumvents problems encountered with antisera exhibiting low affinity against bacteria or cross-reactivity against host structures. We have observed that in such situations, clear discrimination between intracellular and extracellular bacteria by the conventional $\mathrm{AB}$ staining procedure is sometimes difficult as either the intracelluar bacteria are only weakly labelled after cellular permeabilization or background staining of host structures lowers the signal-to-noise ratio (Agerer and Hauck, unpublished observations).

On the other hand, the FBA staining requires a covalent modification of bacterial surface residues. Therefore, the labelling could potentially interfere with normal adhesion of the microorganisms to eukaryotic cells. Although we have not observed the negative effects of FITC and biotin labelling on the invasive capabilities of $N$. gonorrhoeae or $S$. aureus at the FITC and biotin concentrations used in this study, one should be cautious in all cases where such an interference has not been excluded. Clearly, our results show that the method can be applied to Gram-positive as well as Gram-negative bacteria and that it yields nearly identical results when compared to the conventional double cycle $\mathrm{AB}$ staining method.

One further advantage that we observed is the stronger staining intensity of the directly labelled microorganisms, in particular of the intracellular fraction of the bacteria. This might be due to an even labelling of the bacterial population prior to infection during the FBA staining versus a slightly reduced accessibility of intracellular bacteria for antibodies, when they are stained after permeabilization of host cell membranes in the conventional double cycle AB staining procedure. Accordingly, the detection of intracellular bacteria as well as the discrimination between intracellular and extracellular bacteria following FBA staining is very consistent in consecutive experiments and, therefore, is more robust. In addition, to the fluorescent dyes employed for FBA staining in this investigation (FITC and Alexa647), numerous alternative dyes with distinct spectral properties exist. Thus, the FBA staining protocol could be modified using for example reactive rhodamine derivatives to covalently label the bacteria or one of a number of fluorescence-marked avidin varieties to suit every microscope set-up. We achieved the best results when FITC and Alexa647 were used to differentially label the bacteria as the spectra of these fluorescent dyes are so distinct that, despite the strong labelling of the bacteria, a bleed-through of fluorescence signals detected with separate filter sets is virtually absent. It is important to note, however, that samples containing bacteria pre-labelled with FITC should be microscopically analysed as soon as possible, because the FITC label decays significantly when storage (even at $4{ }^{\circ} \mathrm{C}$ in the dark) exceeds more than a week (Agerer and Waeckerle, unpublished observations).

One interesting feature of the novel protocol is the lack of cell permeabilization required to discriminate between extracellular and intracellular bacteria. Usually, the permeabilization of host cell membranes is achieved with one of several detergents, such as saponin, Triton X-100 or cholesterol chelators such as filipin (Dehio et al., 1997; Hauck et al., 1998; Holtta-Vuori et al., 2002). However, our own observations with the conventional double cycle $\mathrm{AB}$ staining point to differences in cell permeabilization as a cause of variation between consecutive experiments (Agerer and Hauck, unpublished observations). As the novel FBA staining protocol does not require cell permeabilization, it eliminates one potential source of experimental variation. Clearly, the labelling of the bacteria prior to infection during FBA staining requires additional handling. However, the preparation of microscope samples by the novel protocol is faster than with previous methods (Fig. 1). Compared to the double cycle $\mathrm{AB}$ staining, FBA staining shaves 
about $50 \%$ of the time required to complete the procedure and this is mostly due to the lack of a second staining cycle. Although most of the time to discriminate between extracellular and intracellular bacteria will still have to be spent at the microscope and/or the computer screen evaluating the stained samples, the faster FBA staining protocol nevertheless allows more samples to be processed in parallel, and reduces the amount of handling and light exposure of valuable probes further contributing to a better quality of the stained samples.

Taken together, the novel staining protocol to differentiate between extracellular and intracellular bacteria described here provides a convenient and reliable alternative to existing staining techniques. Moreover, as this approach is independent of specific antibodies directed against the microorganisms, it opens new avenues to quickly determine the invasive potential of unconventional or emerging pathogens.

\section{Acknowledgements}

We thank T.F. Meyer (MPI für Infektionsbiologie) for the Neisseria strains used in this study, and D. Deininger for expert technical assistance. This study was supported by funds from the BMBF (01 KI 8906/ 0) and DFG (Ha2568/3-1) to C.R.H.

\section{References}

Agerer, F., Michel, A., Ohlsen, K., Hauck, C.R., 2003. Integrinmediated invasion of Staphylococcus aureus into human cells requires Src family protein tyrosine kinases. J. Biol. Chem. 278, 42524-42531.

Billker, O., Popp, A., Brinkmann, V., Wenig, G., Schneider, J., Caron, E., Meyer, T.F., 2002. Distinct mechanisms of internalization of Neisseria gonorrhoeae by members of the CEACAM receptor family involving Rac1- and Cdc42- dependent and independent pathways. EMBO J. 21, 560-571.

Booth, J.W., Telio, D., Liao, E.H., McCaw, S.E., Matsuo, T., Grinstein, S., Gray-Owen, S.D., 2003. Phosphatidylinositol 3kinases in carcinoembryonic antigen-related cellular adhesion molecule-mediated internalization of Neisseria gonorrhoeae. J. Biol. Chem. 278, 14037-14045.

Dehio, C., Meyer, M., Berger, J., Schwarz, H., Lanz, C., 1997. Interaction of Bartonella henselae with endothelial cells results in bacterial aggregation on the cell surface and the subsequent engulfment and internalisation of the bacterial aggregate by a unique structure, the invasome. J. Cell. Sci. 110, 2141-2154.

Drevets, D.A., Campbell, P.A., 1991. Macrophage phagocytosis: use of fluorescence microscopy to distinguish between extracellular and intracellular bacteria. J. Immunol. Methods 142, 31-38.

Drevets, D.A., Elliott, A.M., 1995. Fluorescence labeling of bacteria for studies of intracellular pathogenesis. J. Immunol. Methods $187,69-79$.

Falkow, S., 1991. Bacterial entry into eukaryotic cells. Cell 65, $1099-1102$.

Finlay, B.B., Cossart, P., 1997. Exploitation of mammalian host cell functions by bacterial pathogens. Science 276, 718-725.

Fowler, T., Wann, E.R., Joh, D., Johansson, S., Foster, T.J., Hook, M., 2000. Cellular invasion by Staphylococcus aureus involves a fibronectin bridge between the bacterial fibronectin-binding MSCRAMMs and host cell beta1 integrins. Eur. J. Cell Biol. $79,672-679$.

Hauck, C.R., Meyer, T.F., 2003. 'Small' talk: Opa proteins as mediators of Neisseria-host-cell communication. Curr. Opin. Microbiol. 6, 43-49.

Hauck, C.R., Meyer, T.F., Lang, F., Gulbins, E., 1998. CD66-mediated phagocytosis of $\mathrm{Opa}_{52}$ Neisseria gonorrhoeae requires a Src-like tyrosine kinase- and Rac1- dependent signalling pathway. EMBO J. 17, 443-454.

Heesemann, J., Laufs, R., 1985. Double immunofluorescence microscopic technique for accurate differentiation of extracellularly and intracellularly located bacteria in cell culture. J. Clin. Microbiol. 22, 168-175.

Holtta-Vuori, M., Tanhuanpaa, K., Mobius, W., Somerharju, P., Ikonen, E., 2002. Modulation of cellular cholesterol transport and homeostasis by Rab11. Mol. Biol. Cell 13, 3107-3122.

Luhrmann, A., Streker, K., Schuttfort, A., Daniels, J.J., Haas, A., 2001. Afipia felis induces uptake by macrophages directly into a nonendocytic compartment. Proc. Natl. Acad. Sci. U. S. A. 98, $7271-7276$

McCaw, S.E., Schneider, J., Liao, E.H., Zimmermann, W., GrayOwen, S.D., 2003. Immunoreceptor tyrosine-based activation motif phosphorylation during engulfment of Neisseria gonorrhoeae by the neutrophil-restricted CEACAM3 (CD66d) receptor. Mol. Microbiol. 49, 623-637.

Schmitter, T., Agerer, F., Peterson, L., Muenzner, P., Hauck, C.R., 2004. Granulocyte CEACAM3 is a phagocytic receptor of the innate immune system that mediates recognition and elimination of human-specific pathogens. J. Exp. Med. 199, 35-46.

Sieg, D.J., Hauck, C.R., Schlaepfer, D.D., 1999. Required role of focal adhesion kinase (FAK) for integrin-stimulated cell migration. J. Cell Sci. 112, 2677-2691.

Sinha, B., Francois, P.P., Nusse, O., Foti, M., Hartford, O.M., Vaudaux, P., Foster, T.J., Lew, D.P., Herrmann, M., Krause, K.H., 1999. Fibronectin-binding protein acts as Staphylococcus aureus invasion via fibronectin bridging to integrin alpha5beta1. Cell. Microbiol. 1, 101-117.

Williams, J.M., Chen, G.-C., Zhu, L., Rest, R.F., 1998. Using the yeast two-hybrid system to identify human epithelial cell proteins that bind gonococcal Opa proteins: intracellular gonococci bind pyruvate kinase via their Opa proteins and require host pyruvate for growth. Mol. Microbiol. 27, 171-186. 